\title{
A Satisfactory Science of Mind, and the Connection Between Mind Science and Brain Science
}

\author{
Peter O'Hara, Registrar, Adult Psychiatry, St. Loman's Hospital, Dublin 20
}

In this article, 'mind science' refers to any knowledge derived from the study of behaviour, in which I include selfreports of mental states. 'Brain science' refers to anatomy, physiology, and biochemistry. Psychiatrists have contact with both types of science, especially in helping to construct therapies. Some choose help more from the one or the other, but even those who make use of both rarely see any connection between the two sciences. There are also difficulties over what is truly scientific in the 'mind science' sector. This article looks at these two problems.

\section{A true mind science?}

Just what should be accepted as scientific in 'mind science' is a vexed question. Many people today find difficulty in understanding why psychology at one time took the step of limiting its field to observable behaviour. One needs to remember that until about a hundred years ago psychology was a sub-section of philosophy, just as the physical sciences were two or three hundred years previously. This attitude persisted into the 1950s in at least one university philosophy department. By introspection and reflection, philosophers described such major psychological subjects as perception. The reaction to such writing by some of those who wished to found a scientific psychology went too far in the other direction when they excluded a person's own report of his mental state from consideration as evidence.

That mistake has now been remedied. Many investigations of normal people use their utterances as evidence, but as one element of behaviour, and without presuming them to be true. In psychiatry also, many investigations of epidemiology, psychopathology, and therapy use measurement schemes based on self-reports in whole or in part. The originator of such a instrument usually conducts studies to show that it is reliable and valid. This type of investigation fits the criteria for a scientific 'mind science': firstly, because it tries to control for the variable of the investigator; and secondly, because it only makes statements about groups of people, a requirement based on the large number of confounding variables, most of which are not known to the investigator, and only a few of which can be controlled or eliminated.

\section{The mind-brain connection}

A great vision of a possible mind-brain connection appeared when the function of the neuron was first described. There are ten thousand million neurons in the brain: each summates hundreds or thousands of facilitory and inhibitory inputs to determine its output (whether to fire or not); the output is often distributed to many other cells; such brain machinery is clearly capable of very elaborate functions. However, apart from sensory and motor functions, little has been added to fill out that vision.
The main problem is that we have expected the connection to be too simple. This is based on success in investigating the physiology of other body systems. For example, the kidney is made of about one million nephrons. The nephron is not at a level comparable to the neuron: it is a tubule composed of hundreds of cells. But in that one unit all kidney function is carried out. To achieve the function of one full kidney, you simply multiply by a million. In like manner we have expected to find mental functions written in miniature in small subsections of the brain. One reaction to the failure to find such 'inscriptions' is to abandon the claim that the whole can be explained by the sum of the parts: this means that it is explained by the parts plus something else: and this something is amorphous and cannot be described.

A way out of this difficulty is being shown by Artificial Intelligence investigators. These people have come from psychology and computer science. A clear picture of the mindbrain connection can be found in Hofstadter.' The principle is that the construction of computers, which can carry out some human functions, throws light by analogy on possible mindbrain connections. I will give some examples of these analogies, together with analogies from physical systems.

\section{Levels of description}

Any system can be described at different levels. The basic unit of electronic computers is a junction of two slightly different kinds of silicon, analogous to a transistor. There is one output (either a current passing or not passing) and two or more inputs. A description of the state of a computer at this level could be a list of ones and noughts, one for each silicon junction (of which there may be thousands of millions). This cannot be easily related to the function we see the computer as performing. Similarly, a list of the resting versus spiking state of the ten thousand million neurons in a human brain is not easily related to a mental state description.

The next levels of description are illustrated by computer languages. Instructions in the form of ones and noughts are called machine code: this is directly understood by silicon chips, and was the method used to instruct computers in the early states of development. Such a programme consists of binary numbers, but it can be described in chunks by ordinary language words: this is called assembly language-'add', 'jump', and 'divide' would appear in it. A programme written in machine code is needed to translate the assembly language into machine code. After programmers had used assembly language for some years they noticed patterns in their use of the instructions, and they chunked these into descriptions in higher-level languages, called compiler languages. A programme written in assembly language translates the compiler language into assembly language-this is called the compiler programme. The point to note here is that, while there is a 
simple relation between words in assembly language and numbers in machine code, and also between words in compiler languages and words in assembly language, there is no simple relation between words in compiler language and numbers in machine code. Perhaps ten or twenty or more levels would be needed to relate neuron function to mental state descriptions, and each level could only easily be related to those next to it, or occasionally to the second next level.

A mass of gas can be described by detailing the position, mass and velocity of each molecule at any one instant. This description changes with time. There is also a high-level description of the volume, pressure and temperature of the whole of the gas. These seem far apart, but in fact there is no intermediate level of description between them.

\section{A physicist describes himself}

Let us suppose a nuclear physicist wants to write a description of himself. He knows that all matter is made of quarks and electrons, and so his description will be a list of every such particle in his body and the relations of each to every other particle. While the physicist has not the means to obtain these data, and could only write out a tiny part of it in his own lifetime, it is a theoretically possible description, and it would provide a highly deterministic account of a person's behaviour.

A molecular biologist will suggest a description at the level of molecules. This list will include every molecule, their types, and their relations to every other molecule. This shorter description is still beyond a lifetime's computing power.

A cell biologist will put forward a cell level description. This is shorter again, but not short enough for practical use for the whole person. There are further (higher) levels of description, namely tissues, organs, and body systems. The transitions from one level to the next are already described for most systems except the central nervous system. The final, unitary description of the person bears no simple relationship to the subatomic particle description.

\section{Chunking}

When a low-level description is chunked to produce a highlevel description the great advantage is the saving in length. For example, the cell-level list for a human is much shorter than the molecular description. However, in chunking, determinism is lost, and only a probabilistic description of the system's behaviour can be given. In the mass of gas the molecular description specifies each molecule's position and velocity. In the pressure-volume-temperature description only a probability can be calculated of finding a molecule in a particular space or of a molecule having velocity within a particular range.

\section{Epiphenomena}

An epiphenomenon is a property that is not stored descriptively but is a consequence of the overall system organization. For example, if you divide up a mass of gas smaller and smaller, you find that each part has the same pressure and temperature as the original whole. You might expect this to continue indefinitely, but when the part is one molecule you find that it has no 'temperature' or 'pressure'. It also fails to show a 'volume' corresponding to previous divisions of volume as you move lower down the scale. The pressure, temperature, and volume are not properties of individual molecules. They are parts of the description at a high level.

My final example is particularly intended to illustrate the unexpected qualitative difference between the epiphenomena and the attributes contributing to it in the lower-level description. A computer is programmed to keep a set of square 'cells' in a two-dimensional space: they are appropriately illustrated on a television screen. There are two populations of cells. distinguished by two colours, and initially they are set in two discreet but adjacent areas or 'blobs'. In each of many successive moves each cell can move randomly up, down, left or right. The only difference between the two groups is in 'adhesiveness' to adjoining cells, each group being uniform but one group more 'sticky' than the other. As the programme runs the less adhesive ones spread around the more adhesive ones, and eventually form a uniform ring or 'coat' around the latter. Programmes of this type may be useful in modelling embryology. The high-level description is of one cell type forming a ring around the other, but this is not recorded in each cell: rather a seemingly unrelated property (adhesiveness) is recorded there.

I will now summarize the lessons to be learned from these analogies. Firstly, the connection from neuron function to mental description will not be simple but will involve many intermediate levels. There is no knowledge yet of what kind of chunks of neurons will form the first step up, let alone the higher steps. When higher-level descriptions are produced, it may be possible to write a complete description of the mind; at lower levels the full description will be too long to write out in practice, but there will be a description of the kind of description involved and the features that would appear in it. Secondly, the higher the level of a description, the less certainty about the involvement of individual neurons. Thirdly, and most importantly, we should not expect to find any lowlevel features corresponding to higher mental functions. The high hopes of finding associations between variations in level of neurotransmitters or receptors and major mental changes may be mistaken. The low-level variables which we will find underlying our mental processes may appear quite banal, as in the example of the cellular computer programme.

Finally, to those who feel these analogies are mistaken because computers are rational and minds are irrational or unpredictable, I would say that the problem is of a confusion of levels. Computers are described as rational at the lowest level: each silicon junction behaves predictably. Neurons are also predictable when they add up the facilitatory and inhibitory inputs in determining whether to spike or not. At the higher level computers can be programmed to act irrationally, while remaining fully rational at the bottom level. Our 'irrational' minds have the same relation to our 'rational' neurons.

\section{REFERENCE}

IHofstadter, D. R. (1979) Gödel, Escher, Bach: An Eternal Golden Braid. London: Penguin. 